ESJ Humanities

\title{
Analyse des problèmes liés au parcours juridique des victimes des violences sexuelles dans la province de la Tshopo en République Démocratique du Congo
}

\author{
Augustin Mukiekie Tshite, Professeur \\ Sébastien Loosa Bolamba, Professeur \\ Université de Kisangani, RD. Congo \\ Olivier Schmitz
}

Chercheur, Ottignies-Louvain-la-Neuve, Belgique

An Verest

Chercheuse, Université de Gand, Gand, Bruxelles

Bosmans Marleen

Experte Genre et Droits Humains, Enabel, Bruxelles, Belgique

Edouard Konan

Assistant Technique International, Enabel, Kisangani, RD. Congo

Chantal Nandindo

Assistante Technique Nationale, Enabel, Kisangani, RD. Congo

\section{Doi:10.19044/esj.2021.v17n33p214}

Submitted: 03 August 2021

Accepted: 23 August 2021

Published: 30 September 2021
Copyright 2021 Author(s)

Under Creative Commons BY-NC-ND

4.0 OPEN ACCESS

Cite As:

Tshite A.M., Bolamba S.L., Schmitz O., Verest A., Marleen B., Konan E. \& Nandindo C. (2021). Analyse des problèmes liés au parcours juridique des victimes des violences sexuelles dans la province de la Tshopo en République Démocratique du Congo. European Scientific Journal, ESJ, 17 (33), 214. https://doi.org/10.19044/esj.2021.v17n33p214

\section{Résumé}

Cette étude vise deux objectifs, à savoir : dégager les problèmes liés au parcours juridique des victimes de violences sexuelles dans la province de la Tshopo et, proposer ce qui doit être fait pour élaguer ces problèmes et permettre une prise en charge efficace et équitable. Pour y arriver, l'étude qui s'inscrit dans le contexte de la théorisation ancrée a mobilisé comme sources les dossiers des victimes des violences sexuelles en justice, les comptes rendus des réunions, les groupes de discussion, les récits de vie des victimes et la littérature grise. Partant, il se dégage que les problèmes identifiés sont multiples et de diverses natures. On répertorie notamment: le dysfonctionnement des services judiciaires ; l'absence des preuves par des 
parties concernées (médecins, police, agents pénitentiaires, etc.); une pauvreté extrême des victimes ; les manœuvres dilatoires tendant à repousser perpétuellement les audiences pour freiner le procès ou bloquer l'action par des procédures irrégulières ; l'arrangement à l'amiable ; l'insuffisance et l'éloignement géographique des juridictions compétentes en matière de violence sexuelle ; l'environnement social des victimes; et l'insécurité et la crainte de représailles. Que faire? Le problème des violences sexuelles nécessite l'implication de toutes les communautés. Aussi, les campagnes de sensibilisation, les sessions de formations ainsi que des actions concrètes qui pourraient apporter des solutions aux problèmes répertoriés.

Mots-clés: Violence, violences sexuelles, parcours juridique, problèmes, Tshopo

\title{
Analysis of the Problems Linked to the Legal Course of Victims of Sexual Violence in the Province of Tshopo in the Democratic Republic of Congo
}

\author{
Augustin Mukiekie Tshite, Professeur \\ Sébastien Loosa Bolamba, Professeur \\ Université de Kisangani, RD. Congo \\ Olivier Schmitz
}

Chercheur, Ottignies-Louvain-la-Neuve, Belgique

An Verest

Chercheuse, Université de Gand, Gand, Bruxelles

\section{Bosmans Marleen}

Experte Genre et Droits Humains, Enabel, Bruxelles, Belgique

Edouard Konan

Assistant Technique International, Enabel, Kisangani, RD. Congo

Chantal Nandindo

Assistante Technique Nationale, Enabel, Kisangani, RD. Congo

\begin{abstract}
This study has a dual objective which firstly aims to identify the problems associated with the legal process of victims of sexual violence in the province of Tshopo, and followingly to share recommendations to eliminate the identified problems to ensure that victims' are dealt with effectively and fairly along the criminal proceedings. To achieve this, the study, using a Grounded Theory methodology, used the files of victims of sexual violence in the courts, the minutes of meetings, discussion groups, the life stories of
\end{abstract}


victims and grey literature as sources. The problems identified are multiple and varied and include: dysfunctional judicial services; lack of evidence from the parties concerned (doctors, police, prison officers, etc.); extreme poverty of the victims; delaying tactics that tend to perpetually postpone hearings in order to slow down the trial or block the action through irregular procedures; out-of-court settlements; the inadequacy and geographical remoteness of the jurisdictions competent in matters of sexual violence; the social environment of the victims; and insecurity and fear of reprisals. What can be done? The problem of sexual violence requires the involvement of the whole community. Given that the problem of sexual violence requires the involvement of the whole community, this research proposes awareness-raising campaigns, training sessions and concrete actions as solutions to the problems identified.

Keywords: Violence, sexual violence, legal course, problems, Tshopo

\section{Introduction}

Pendant longtemps, les gens ne voyaient aucun problème dans le comportement sexuel, présenté comme la manifestation d'un instinct monolithique, celui de la propagation de l'espèce (Duyckaerts, 1964). Toutefois, à côté de cette conception classique de la sexualité, aujourd'hui les victimes des violences sexuelles sont confrontées à plusieurs problèmes qui constituent un handicap majeur à leur parcours juridique. A cet effet, les acteurs des différents domaines (juriste, politologue, sociologue, psychologue, anthropologue, médecin,...) sont mobilisés pour la cause des victimes des violences sexuelles dans ce parcours.

Cet intérêt orienté vers les problèmes liés aux violences sexuelles pour ces différents acteurs précités, paraît être justifié par l'ampleur qu'ils ne cessent de prendre au fil des années en termes de persistance, de nombre de victimes voire des multiples conséquences collatérales négatives, notamment de points de vue psychologique, économique et social pour les victimes. Ainsi, en raison de leur ampleur, ces dernières années, les violences sexuelles font l'objet d'une attention soutenue, aussi bien des différents gouvernements que des organisations non gouvernementales, des mouvements féministes et même par des chercheurs.

Dans le monde, (OMS et al., 2021) ont affirmé, dans un communiqué, que la violence à l'égard des femmes reste terriblement omniprésente et débute très tôt dans la vie d'une femme, comme le montrent de nouvelles données alarmantes publiées par l'OMS et ses partenaires. Au cours de sa vie, d'après le communiqué, une femme sur trois est victime de violence physique ou sexuelle de la part d'un partenaire intime ou de violence sexuelle de la part de quelqu'un d'autre que son partenaire, soit environ 736 millions de femmes, un 
nombre qui est resté en grande partie inchangé au cours de la dernière décennie.

Ceci est une raison pouvant justifier la mobilisation actuelle des différents dirigeants et partenaires pour tenter d'éradiquer ce phénomène dont les conséquences pour les victimes sont incalculables. Malgré cette mobilisation, l'ampleur du phénomène ne faiblit pas, bien au contraire. À titre illustratif, CVS, INSSEE-ONDRP (2013) présentent l'exemple de la France où une femme sur 5 et un homme sur 14 rapportent avoir été victimes d'agressions sexuelles au moins une fois dans leur vie. La même enquête rapporte que chaque année, 102.000 personnes adultes de 18 à 75 ans, dont 86.000 Femmes et 16.000 hommes, sont victimes de viol ou des tentatives de viol en France métropolitaine.

La situation en Afrique semble être plus difficile et complexe car, comme l'indique Ombiabo (2020), il est difficile de chiffrer la problématique des violences sexuelles avec exactitude sur le continent. Plusieurs sources (la police, les instances judiciaires, les services médicaux, les organisations non gouvernementales, les associations des femmes pour la lutte contre les violences sexuelles s'accordent à dire que celles-ci sont dues aux conflits armées. C'est particulièrement le cas en RD. Congo, pays auquel s'intéresse spécifiquement la présente recherche.

La RD. Congo fait malheureusement partie des pays qui payent le lourd tribut des violences sexuelles, avec de milliers des cas rapportés chaque année, notamment dans sa partie Est. Les guerres, les conflits armés récurrents dans cette partie du pays, ainsi que l'insécurité et le climat d'impunité qui y règne, notamment du fait de la persistance des groupes armés incontrôlés et des poches d'insécurité, sont autant des facteurs qui ont fait et qui continuent à faire le lit de cette pire forme de violation des droits humains (UNFPA, 2013).

Pour montrer la complexité des violences sexuelles en RD. Congo, Maertens de Noordhout (2013) affirme qu'elles sont particulièrement difficiles à combattre parce qu'elles sont largement répandues et qu'elles ne sont pas cantonnées à un groupe déterminé. L'auteur a regroupé les agresseurs en trois grandes catégories, à savoir 1) les milices rebelles, 2) les Forces armées de la RD. Congo et les officiers de la Police Nationale congolaise et, 3) les civils eux-mêmes. Ceci signifie que si hier les violences sexuelles étaient généralement attribuées aux premières catégories citées, la situation a beaucoup changé aujourd'hui. À cet effet, dans un rapport publié par l'UNFPA (Vasseur et al., 2019), il est clairement montré que parmi les viols commis entre 2005-2017 en RD. Congo, 58\% l'ont été par les civils et les combattants, $8 \%$ par les militaires ou policiers et $34 \%$ par les miliciens.

Une autre étude menée par Peterman et al. (2011) rapporte que jusqu'à 1,8 million de femmes congolaises ont été violées au moins une fois dans leur 
vie. Partant de toutes ces statistiques, il y a lieu de reconnaitre que l'ampleur du phénomène est inquiétante. Les femmes adultes, comme les enfants sont violés en RD. Congo. Tremblay (2005) fait observer que l'agression sexuelle commise envers les enfants constitue un phénomène inhérent à notre société, qui existe et perdure depuis longtemps à travers de nombreuses générations.

De ce qui précède, il se dégage que les statistiques concernant l'ampleur des violences sexuelles sont alarmantes. Cet état de choses justifie actuellement la multiplicité de recherches tendant à mettre en place des mécanismes à exploiter pour contrer la propagation (l'expansion) des violences sexuelles, tout en sanctionnant sévèrement les auteurs et en protégeant les victimes. C'est dans ce contexte que les textes sont produits, les lois sont votées pour définir le cadre conceptuel et les mesures pratiques à mettre en œuvre toutes les fois que des actes relatifs aux violences sexuelles sont commis. À cet effet, les communautés en général et les femmes violées en particulier sont sensibilisées à saisir la justice, les formations sont assurées, des structures de protection et d'accompagnement des victimes sont créées, des recherches sont initiées pour identifier les causes, publier les statistiques, apprécier le vécu et la prise en charge des victimes des violences sexuelles.

En dépit de toutes les sensibilisations à saisir la justice par les victimes des violences sexuelles, à Kisangani où la présenté étude est menée, au cours d'un atelier organisé par l'ONG Centre de Documentation pour la Femme, en partenariat avec la Commission Nationale de Droit de l'Homme et la Magistrature Civile, (CDF, 2017), il a été constaté qu'il y a trop de problèmes d'arrangements à l'amiable et que la population ne dénonce pas les violences faites par crainte des représailles. Ainsi, les victimes des violences sexuelles ont été encouragées à saisir la justice pour obtenir réparation, nonobstant les problèmes liés à cette dénonciation.

Malgré tous ces efforts et en dépit de l'architecture juridique qui encadre les questions des violences sexuelles, les victimes ne dénoncent pas (CDF, 2017), prétextant qu'elles rencontrent plusieurs problèmes de nature à entraver le parcours juridique de leurs dossiers (instruction juridique) et prise en charge, entre autres. C'est dans cette veine que la société civile (Société civile, 2019) a souligné dans sa déclaration que la violence sexuelle est un sujet difficile à aborder et souvent mal compris. En général, poursuit-elle, les lois et les politiques y relatives ne reflètent pas la réalité de l'expérience vécue par les victimes et les efforts en matière de responsabilisation ne donnent que peu de résultats. Ce constat est souvent observé dans la Province de la Tshopo en ce qui concerne le traitement ou la prise en charge judiciaire des cas des violences sexuelles. Concrètement, les tracasseries décriées des services pour différents frais exigés, la lenteur dans l'instruction des dossiers, des stigmatisations de divers ordres, des discriminations, des difficultés à rassembler des preuves constituent le tableau sombre susceptible d'attirer 
l'attention des personnes intéressées par cette thématique ou de tout observateur curieux. Ce constat justifie le bien-fondé de cette étude qui est menée sous l'égide de l'Agence Belge de Développement (Enabel), en partenariat avec l'Université Catholique de Louvain (UCL) et l'Université de Gand (UGENT). Aussi, cette étude se justifie-t-elle par le souci de mettre en lumière, sur base d'une analyse minutieuse des processus d'instruction des dossiers de cas des violences sexuelles, les différents problèmes rencontrés par les victimes.

Pour ce faire, l'étude exploite les récits des victimes compulsés à travers de différentes structures tant judiciaires que de la Police de la Ville de Kisangani, les contenus des échanges avec certaines victimes et leurs proches, les échanges avec des différents membres et de personnalités de la communauté (leaders), avec pour visée la possibilité que les résultats de cette étude pourraient aider à faire des plaidoiries bien ciblées et bien soutenues pour une justice plus satisfaisante. Elle se donne pour objectif d'apporter des réponses aux questions ci-après :

- Quels sont les différents problèmes que rencontrent les victimes des violences sexuelles tout au long de l'instruction en justice de leurs dossiers ?

- Quelles stratégies peut-on déployer pour élaguer ces problèmes et permettre une prise en charge efficace et équitable des victimes de ces violences?

\section{Méthodologie de la recherche}

\section{A. Contexte, population et échantillon de l'étude}

Cette est menée dans la ville de Kisangani, chef-lieu de la province de la Tshopo en RD. Congo. Depuis 2018 à Kisangani, l'Agence Belge de Développement, Enabel-PLVS (Programme de Lutte contre les Violences Sexuelles) en partenariat avec les universités Catholique de Louvain (UCL), de Gand (UGENT) et de Kisangani (UNIKIS), y a initié une recherche-action au Centre de Santé Alwaleed, qui utilise une approche multisectorielle comprenant une assistance médicale, psychosociale, juridique et socioéconomique en faveur des victimes de viol.

Cette étude est menée auprès des victimes rétablies, suivies par le Centre Alwaleed dans la ville de Kisangani en RD. Congo. Étant donné que certaines victimes des violences sexuelles ne fréquentent, ni le Centre de prise en charge psychosociale et médicale (Centre Alwaleed), ni la justice pour le suivi de leurs dossiers, il est difficile d'accéder, au niveau des structures étatiques à des statistiques y relatives. Ainsi, cette recherche ne prend en considération que les victimes des violences sexuelles identifiées; celles qui ont été prises en charge au Centre Prince Alwaleed de Kisangani en l'occurrence. D'après les statistiques recueillies dans ledit centre pour une 
période de 5 ans (2015-2020), période où les statistiques ont été rendues disponibles grâce à l'accompagnement financier d'Enabel, l'effectif des victimes des violences sexuelles ayant fréquenté le Centre Alwaleed pour une prise en charge est allé croissant, comme le révèle la figure 1:

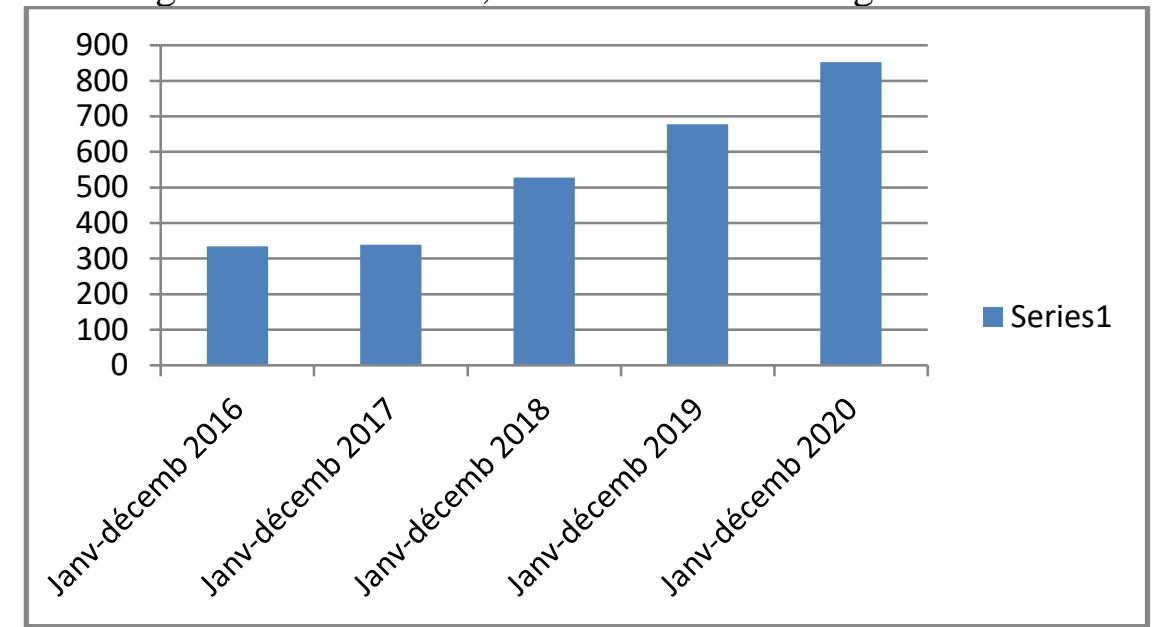

Figure 1. Effectifs des victimes prises en charge au Centre Alwaleed de 2016 à 2020

Considérant les données de la figure 1. ci-dessus, il se dégage que l'effectif total des victimes des violences sexuelles ayant consulté le Centre Alwaleed, pour cette période de cinq ans, est de 2.732 sujets. L'année 2020 a le nombre le plus élevé des victimes. Elle représente à elle seule une proportion de $31,22 \%$, soit plus de deux quarts de l'ensemble des victimes. Ainsi, par rapport à l'évolution des cas pris en charge par le Centre Alwaleed, il s'observe que la courbe est ascendante. Ceci veut dire que l'effectif ne cesse d'augmenter au fil des années retenues.

En raison du fait que, d'une part, que tous les dossiers de ces 2.732 victimes suivies par le Centre Alwaleed ne sont passés par la justice et que, d'autre part, la conservation des archives en justice est un casse-tête ne permettant pas d'en retrouver les traces, la présente recherche est réalisée sur un échantillon de 30 dossiers tirés de l'ensemble, du fait de leur traçabilité, après des démarches auprès des archives des Tribunaux pour Enfant, Lutte Contre les Violences Sexuelles et de Grande Instance de Kisangani. Les analyses desdits dossiers sont complétées par les informations issues de diverses sources ou matériaux, à savoir les comptes rendus des réunions et les discussions sur les violences sexuelles, les archives des structures de prise en charge des victimes des violences sexuelles, l'écoute auprès des victimes et de leurs proches, ainsi que les entretiens individuels. Toutes ces sources sont décrites dans la section relative à la collecte des matériaux.

Étant donné que cette étude a eu recours à l'approche dite « Grounded theory », les victimes des violences sexuelles, les leaders communautaires, les 
dossiers partis du Centre Alwaleed vers la justice, les entretiens avec les victimes et leurs proches, les structures de prise en charge des victimes des violences sexuelles constituent l'échantillon théorique. Dans ce contexte, ces matériaux permettent de construire une explication théorique du phénomène étudié. Ils permettront de dégager les différents problèmes rencontrés par les victimes des violences sexuelles. La visée n'est pas de généraliser les découvertes à des populations plus vastes que celles considérées dans cette recherche, mais de comprendre et produire des connaissances en fonction des réalités du milieu.

\section{B. Approche et collecte des matériaux \\ B.1. Approche utilisée}

Cette étude a recouru à une théorie que les Anglo-Saxons appellent «Grounded Theory» (GT) et que les francophones traduisent par: « théorisation ancrée ou enracinée ». C'est une méthode inductive développée par Glaser et Strauss (1967). Son but est de construire une théorie à partir des données, selon une approche où les données sont simultanément recueillies et analysées. Dans sa première version, elle se voulait rattachée au courant positiviste, en offrant une base solide, systématique et claire pour l'analyse des données et la construction de théories (Bryant \& Charmaz, 2007).

Au fil des années, Glaser et Strauss ont pris des chemins divergents quant à leur interprétation de la théorisation ancrée. En effet, leur position initiale soutenait que la réalité est indépendante du chercheur et des méthodes employées pour l'étudier, que l'on peut construire une théorie suffisamment générale pour être applicable à diverses situations et populations (Glaser \& Strauss, 1967). La GT procède autrement : le cadre théorique est construit au contact des données. Ceci veut dire que le chercheur qui utilise la GT doit se défaire des théories, des idées préconçues, des hypothèses formulées sur un problème de société donné. Le chercheur doit affronter la réalité à travers les données récoltées, faire parler ces données en les analysant, en les comparant et de fil en aiguille se faire une idée du problème, une théorie explicative qu'il se construit tout au long de l'enquête.

La GT est une approche empirico-inductive. Elle implique pour le chercheur d'adopter une attitude compréhensive, respectueuse vis-à-vis des données, des informations (aucune donnée n'est mauvaise en soi) et de l'éthique (respect de la personne qui donne l'information; ne pas faire apparaître un signe de rejet, de dénigrement à son égard). Pour le cas de la GT, les éléments explicatifs du phénomène (les théories) sont bien à l'état brut dans le matériau et, c'est au chercheur de les mettre à jour (par interprétation des données recueillies). En raison de l'utilisation de la TG, l'étude a exécuté trois activités, à savoir : 1) identifier les données (matériaux) et les collecter ; 2) analyser les données; et 3) les interpréter afin de les intégrer dans un cadre 
théorique qui se construit, permettant d'améliorer la connaissance et la compréhension du phénomène des violences sexuelles à l'étude.

De l'avis de Marie-Claude et al. (2014), ce qui fait la force de la TG est à situer à trois niveaux, à savoir : 1) elle comporte des étapes d'analyse détaillées qui contribuent à sa rigueur ; 2) elle vise à examiner en profondeur les processus, ce qui permet de mettre en lumière les relations entre les problématiques et les structures sociales ; et enfin, 3) elle procure un langage commun qui aide les acteurs intéressés à mieux comprendre un phénomène et à permettre la mise en œuvre des actions. Ainsi dit, la TG nous a donné la possibilité d'examiner en profondeur les problèmes rencontrés par les victimes des violences sexuelles dans la province de la Tshopo, permettant ainsi d'envisager des actions pour aider les victimes des violences sexuelles. Dans cette veine, 'étude peut réaliser une interprétation la plus plausible des matériaux analysés concernant les problèmes rencontrés par les victimes des violences sexuelles, comme l'ont montré Corbin et Strauss (2008).

La «grounded theory» favorise l'innovation à partir d'un certain nombre de procédures méthodologiques. Guillemette (2006) en cite quatre qui sont reprises par d'autres auteurs. C'est notamment: 1) la suspension temporaire du recours à des cadres théoriques existants (Glaser, 1998 ; 1995, Glaser \& Srauss, 1967) ; 2) la façon particulière de préciser l'objet de recherche (Beck, 1999; Dey,1999; Glaser, 2001 ; May, 1986 ; Strauss \& Corbin, 1998 ; Willig, 2001) ; 3) l'interaction circulaire entre la collecte et l'analyse des données (Mose et Richards, 2002 ; Norton, 1999), et 4) les procédures d'analyse favorisant l'ouverture à l'émergence ou l'écoute des données (Srauss \& Corbin, 1998). La présente étude a tenu compte de ces démarches. À cet effet, l'étude n'a recouru à aucun cadre théorique existant. L'objet de recherche émerge des matériaux collectés. Le va-et-vient dans les matériaux est un indicateur déterminant de l'interaction circulaire.

\section{B.2. Collecte des matériaux}

Les matériaux ayant conduit aux principaux résultats présentés dans la section suivante viennent de plusieurs sources, notamment l'analyse de 30 dossiers des victimes des violences sexuelles partis du Centre Alwaleed vers la justice. Au-delà des dossiers des victimes analysés, l'étude a pris en compte plusieurs autres sources, c'est le cas :

- des Comptes rendus des réunions discussion « focus group ». Au total, 9 focus groups ont été organisés sur différentes thématiques des violences sexuelles (des questions essentiellement ouvertes). Y participaient : des garons et les filles mineurs de moins de 20 ans (élèves du secondaire), des prestataires de soins de santé, des leaders religieux et communautaires (milieux urbain et rural), des hommes et des femmes de plus de 20 ans (milieux rural et urbain), ainsi que les jeunes membres 
des bandes ou de gangs ayant déjà été auteurs d'un ou des plusieurs viols ;

- des comptes rendus du GTM (Groupe de Travail Multisectoriel), un groupe composé d'un sociologue, d'un anthropologue, d'un psychologue, d'un juriste, d'un pédagogue, d'un médecin, d'un politologue, de leaders communautaires, d'une femme ménagère (une victime rétablie), d'un membre de la Division des Affaires Sociales (DIVAS), d'un représentant des groupes de jeunes gangs, et d'un enseignant de la Faculté de Psychologie et des Sciences de l'Éducation de l'Université de Kisangani considéré comme personne ressource en raison de son intérêt pour les thématiques liées aux violences sexuelles. Ce groupe a été constitué en vue de discuter des problèmes-hypothèses soulevés après l'analyse des matériaux (dossiers des victimes des violences sexuelles, récits de vie, ateliers, rapports, documents divers, etc.). Les échanges entre les membres du GTM ont tourné autour des thématiques relatives aux violences sexuelles soulevées pendant l'analyse des matériaux. Au total, dix réunions discussions ont été organisées entre les années 2018 et 2019 ;

- du compte-rendu d'un atelier sur le cadre juridique des violences sexuelles en R D. Congo. Cet atelier avait réuni : 1) quatre enseignantschercheurs de la Faculté de Psychologie et des Sciences de l'Éducation de l'Université de Kisangani ; 2) six magistrats ; 3) trois défenseurs des Droits de l'homme qui travaillent sur les questions des violences sexuelles, tous avocats; 4) deux personnels (un médecin et un psychologue) du Centre Alwaleed ; 5) un officier de la Police de Prévention et Lutte contre les Violences Sexuelles; et 6) deux gestionnaires du programme PLVS/Enabel.

- des archives des structures de prise en charge des violences sexuelles (rapports et autres documents) et les travaux de fin de cycle de graduat et de licence des étudiants de la Faculté de Psychologie et des Sciences de l'Éducation de l'Université de Kisangani ;

- de l'écoute auprès des victimes des violences sexuelles et de leurs proches par les entretiens individuels (récits de vie). L'étude a analysé au total 87 récits de vie, dont 41 avec les victimes « rétablies » et 46 avec les proches des victimes ;

- des entretiens individuels (6) avec les participants des focus groups identifiés comme personnes ressources (1 garçon mineur, 1 fille mineure, 1 homme adulte, 1 prestataire de Santé, 1 leader religieux, 1 membre de gang). 


\section{B.3. Analyse des matériaux}

L'analyse des matériaux s'est déroulée dans le strict respect du principe de circularité de la théorisation ancrée, tel que décrit dans la littérature existante (Charmaz, 2006; Corbin \& Strauss, 2008; Luckerhoff \& Guillemette, 2013; Strauss \& Corbin, 1998). Ceci est bien illustré à travers plusieurs va-etvient dans les récits des victimes de violences sexuelles et leurs proches, les comptes rendus, les transcriptions des discussions de groups (focus groups) et des entretiens. Les discussions des focus groups, riches de sens, ont permis de générer de nouvelles idées ou des hypothèses qui ont fait l'objet de discussions entre les membres du groupe de Travail Multisectoriel (GTM).

L'étude a procédé à une comparaison constante de divers matériaux. Dans cette veine, les analyses des récits de vie et les dossiers des victimes des violences sexuelles ont été confrontés à celles des différentes sources décrites ci-haut, en vue de dégager les ressemblances et les différences éventuelles. Cette comparaison, au cœur de l'analyse dans la théorisation ancrée (Jacques et al., 2014), a permis de dégager les problèmes émergents chez les victimes des violences sexuelles. Ainsi, la crédibilité des résultats est assurée, comme l'indique la littérature existante (Charmaz, 2006 ; Laperrière, 1997; Strauss \& Corbin, 1998).

\section{Présentation des résultats}

Les matériaux analysés révèlent que les victimes des violences sexuelles sont confrontées aux problèmes des diverses natures. Pour cette étude, ces problèmes sont regroupés en deux grandes catégories, il s'agit des problèmes d'ordre général et des problèmes liés au dysfonctionnement de la justice.

\section{C1. Problème d'ordre général}

Ce regroupement comprend globalement cinq problèmes identifiés à la fin de cette étude. Il s'agit de la pauvreté des victimes et de l'ignorance du droit, de l'insécurité et de la peur des représailles, de statut de la femme dans les coutumes, l'atteinte à la dignité de la victime ainsi que de l'environnement social dans lequel vivent les victimes des violences sexuelles.

Considérant la pauvreté des victimes et l'ignorance du droit, la recherche a permis de comprendre qu'au plan économique, la situation des femmes congolaises en général, celle de la Tshopo en particulier, est caractérisée par une extrême pauvreté. Leur condition économique a été fragilisée bien avant les guerres par le dysfonctionnement des structures étatiques et l'absence des infrastructures économiques et sociales viables. Elles sont confrontées au paiement irrégulier des salaires de leurs maris qui travaillent dans la Fonction Publique et les entreprises étatiques. Par conséquent, pour assurer la survie de leurs familles, elles ont dû s'investir dans 
l'agriculture, l'élevage et le secteur informel, à savoir : le petit commerce, la couture, la teinturerie, elles fréquentent les brousses et les forêts pour couper les bois à vendre, la poterie et la vannerie...Ceci est bien illustré par la déclaration d'une victime (18-TSH-KAB-SS-ALW-0058-01-PS): "vers $20 h 00$, elle revenait vendre les maïs sur la route. Du coup, elle a vu 2 garons, l'un de petite taille et l'autre mince et géant. Ils l'ont prise de force, ils ont couché avec elle ». Ainsi, en pratiquant ces activités pour la survie, les femmes sont exposées à la violence sexuelle. On peut donc dire que la pauvreté est un problème majeur pour le parcours juridique des victimes de violences sexuelles, non seulement parce qu'elle les expose aux violences sexuelles, mais aussi parce qu'elle ne leur permet pas de payer les frais occasionnés par la justice. Elles ne peuvent donc pas accéder à la justice pour obtenir réparation.

La présente étude atteste qu'il existe d'autres types de frais engendrés par toute action judiciaire dont il est difficile de s'acquitter pour les victimes. En effet, l'accès au tribunal nécessite de prendre en charge, à maintes reprises les frais de transport du fait des renvois répétés des audiences. Les analyses ont également montré que pour les victimes éloignées des juridictions compétentes et qui doivent effectuer des longues distances, il se pose un problème de frais de séjour lorsque celles-ci doivent se déplacer. Dans ce contexte de paupérisation, il est facile d'imaginer les difficultés financières qu'ont les femmes à avoir accès aux tribunaux. Si la victime a décidé de porter plainte en justice, elle doit payer divers coûts et frais, y compris les frais de justice, les coûts de transport, des analyses et des réquisitions médicales, ainsi que les coûts d'hébergement dans la ville où est situé le tribunal (BCNUD, 2014).

Ces résultats obtenus dans cette étude et ceux de (BCNUDH, 2014 ; Clifford et al., 2008; FOCOBIDERCO, 2019; Ordonez, 2007) sont concordants. Pour ces études, n'accèdent à la Justice en République Démocratique du Congo que les victimes qui ont les moyens financiers et n'en sort satisfaites que celles qui en tirent profit.

Au-delà de la pauvreté, l'étude a permis de se rendre compte que l'ignorance ne facilite pas aux victimes l'accès aux tribunaux. En effet, plusieurs femmes de la Tshopo, sous le joug de la coutume, ignorent leurs droits et ne connaissent pas toutes les possibilités qui leur sont offertes par la justice. Dans un rapport spécial traitant des violences sexuelles en RD. Congo, Lisa et al. (2008), s'inspirant d'une étude menée par la Berkeley-Tulane Initiative on Vulnerable Population et le Centre international pour la justice transitionnelle ont montré que seuls $27 \%$ des gens dans l'est de la RD. Congo et $28 \%$ à Kinshasa sont conscients de l'existence de la Cour Pénale Internationale. Beaucoup de victimes n'ont donc pas connaissance de cette haute juridiction internationale qui juge des crimes de guerre comprenant les 
violences sexuelles. Il y a lieu d'affirmer, à ce stade qu'il se pose un problème de non vulgarisation du droit. Pire encore, l'étude permet de comprendre que certains acteurs du secteur de la justice tels que les Officiers de Police Judiciaire, ainsi que les autorités politico-administratives et coutumières ignorent également les lois édictées à cet effet. Cette ignorance du droit par la victime et certains acteurs entraîne, le plus souvent la saisie des autorités incompétentes.

Un autre problème regroupé dans cette catégorie est l'insécurité et la peur des représailles. Les résultats de cette étude ont montré que les victimes sont exposées à plusieurs exactions. À la Tshopo par exemple, les membres de certains gangs (Kata Moto, États-Unis, Bourgeois, VANDOM, Kossovo, Mexicains, Zaïrois, etc) violent impunément les droits de l'homme et menacent les populations de représailles dans le cas où ces dernières venaient d'une part à collaborer avec leurs ennemis (policiers, militaires, agents de justice) et d'autre part à dénoncer ou assigner en Justice l'un de leurs membres dans les cas des violences sexuelles. Ainsi, les victimes assoiffées de justice ont peur des représailles pour quitter leurs villages et traverser les zones d'insécurité à de longues distances pour atteindre les juridictions en majorité éloignées de leurs domiciles. Celles qui arrivent à saisir la Justice rencontrent leurs agresseurs une fois de retour chez elles et, faute de protection, elles sont l'objet des représailles. Il en est de même lorsque l'agresseur condamné s'échappe de la prison et va retrouver sa victime. Il multiplie alors les stratégies pour se venger. Il en découle que les victimes confrontées à ces des situations hésitent à saisir la Justice. Pour se protéger et protéger les leurs, elles adoptent une attitude consistant à se taire ou à trouver les solutions locales évoquées plus haut.

Le statut de la femme dans les coutumes est un troisième problème dans ce regroupement. En effet, les coutumes de la RD. Congo qui, pour certaines, ont subi l'influence arabo-musulmane, considèrent la femme comme un être faible qui ne peut revendiquer ses droits. Du point de vue sexuel, elle est considérée comme sacrée. Il est interdit de découvrir sa nudité. Une femme dont la nudité a été découverte en garde des blessures intérieures dont toute réparation s'avère difficile. À cet effet, il faut comprendre pourquoi une femme violée préfère se taire car la divulgation d'une telle information jetterait le discrédit sur elle, sa famille ou son foyer. Une jeune fille non mariée verrait ses chances de mariage complètement amoindries, tandis que la femme mariée risquerait de perdre son mari qui la répudierait ou lui reprocherait de « s'être laissée violer ».

Le statut d'être inférieur assigné à la femme par les coutumes ne lui permet pas de faire valoir ses droits en Justice. Par exemple, il n'est pas permis à la femme ménagère de s'absenter pour se rendre à un procès pour une longue période, d'où le fait que la question est généralement réglée par la coutume 
sous la directive des personnes de sexe masculin. La victime a rarement droit à la parole et doit se contenter de l'arrangement fait entre les familles, à savoir le paiement d'une amende dont la nature est fixée par la coutume et que l'agresseur est censé payer. Cette dernière peut être constituée de chèvres et d'argent liquide à remettre à la famille de la victime. Les familles peuvent également s'entendre et pousser l'agresseur à prendre la victime comme épouse.

L'atteinte à la dignité de la victime est le quatrième problème identifié dans ce regroupement. A cet effet, l'étude permet d'observer que les victimes des violences sexuelles ont du mal à déclarer ce qui leur est arrivé, car ceci est un affront qui leur a été fait et qui porte atteinte à leur dignité. Recourir aux Cours et Tribunaux signifierait pour elles exposer leur intimité et raconter à tous les atrocités qui leur ont été infligées. Cet acte n'est pas facile, ce d'autant plus que pour les coutumes, le sexe est un sujet tabou. Dans le même ordre d'idées, il faut mentionner que l'idée d'affronter les multiples visages du public lors d'un procès effraie la femme (la victime) qui appréhende un procès public, en raison de son potentiel destructeur pour sa réputation.

Il est à noter que les nouvelles lois sur les violences sexuelles autorisent le juge à déclarer un huis-clos à la demande du Ministère Public ou de la victime. Il existe aussi le système de codification et d'habillement de façon à cacher la personne qui parle et changer sa voix. Malheureusement, faute d'information sur ces nouvelles lois et dispositifs, certains juges continuent de ne pas décréter un huis-clos. Ils invoquent comme raison à ce refus la nécessité que tout le monde suive la procédure pour dissuader le public de commettre de tels actes. Cette attitude incompréhensible du juge qui viole la loi ferme le prétoire aux femmes désirant garder leur dignité.

Quant à l'environnement social de la femme comme un autre problème, il peut également constituer un frein d'accès à la justice. En effet, les femmes rurales connaissent des difficultés à accéder à la justice à cause des milieux sociaux dans lesquels elles vivent et de nombreux stéréotypes qui les accompagnent au quotidien. Les activités de survie leur prennent la majeure partie de leur temps. Elles éprouvent des difficultés à quitter leur foyer pour effectuer de longues distances afin d'atteindre les Cours et Tribunaux et se résignent à vivre avec leurs blessures. L'étude a révélé que ceci n'est pas toujours le cas pour la femme urbaine. Cette dernière, possédant une certaine instruction, accède à l'information et connaît l'attitude à adopter lorsqu'elle est victime de viol sexuel. Ceci la rend plus apte à saisir les Cours et Tribunaux situés non loin de son domicile. En outre, les organisations non gouvernementales tant locales qu'internationales qui pullulent dans son milieu viennent à son aide pour la défense de ses droits.

Outre la difficulté d'accéder à la justice, l'étude a également montré que les femmes victimes sont objet de rejet dans leurs milieux d'origine. Ceci 
est relevé dans le rapport du CICR (2009) qui a montré qu'entre 10\% et 15\% des victimes identifiées via les maisons d'écoute soutenues par le CICR font face à des problèmes de rejet. Pour favoriser leur réintégration, le CICR (2009) propose de reconstruire en priorité l'estime de soi et le sentiment d'indispensabilité de chaque victime dans sa propre communauté. Ces personnes ont été humiliées, atteintes au plus profond de leur dignité et ont souvent le sentiment d'être seules face à ces souffrances. Cette solitude peut les entrainer dans une spirale de rupture de la communication et des relations sociales.

\section{C2. Dysfonctionnement de l'appareil judiciaire}

A ce niveau, six problèmes sont regroupés et constituent un handicap majeur au parcours juridique des victimes des violences sexuelles. L'étude a révélé le dysfonctionnement de l'appareil judiciaire, l'insuffisance et l'éloignement géographique des juridictions destinées à instruire les dossiers des violences sexuelles, la plainte déposée contre les inconnus, le manque de confiance à la justice par les victimes et leurs familles, l'établissement des preuves par le personnel de la justice ainsi que les problèmes relatifs à la poursuite des agresseurs par les instances judiciaires. Tous ces problèmes sont décrits dans les lignes qui suivent.

Le dysfonctionnement de l'appareil judiciaire entraine ainsi un recours fréquent au règlement à l'amiable ou coutumier. L'étude a révélé que la justice de la Tshopo est sujette, de façon générale, à un dysfonctionnement dont les causes sont nombreuses. On peut répertorier, entre autres, la lenteur excessive du système judiciaire, le report des audiences, le coût élevé des frais en justice, les mauvaises conditions de travail des agents engagés dans ce service, le mauvais traitement salarial des magistrats, le manque des infrastructures et des ressources en quantité suffisante, etc. Ces résultats liés au dysfonctionnement de l'appareil judiciaire que cette étude a permis d'identifier rejoignent ceux du Bureau Conjoint des Nations Unies aux Droits de l'Homme (BCNUDH, 2014). Celui-ci avait révélé comme caractéristiques de la justice en RD. Congo le manque criant des ressources et des capacités, de nombreuses lacunes dans son fonctionnement, le manque des infrastructures et des ressources, le nombre insuffisant des magistrats, le manque des tribunaux et/ou des autorités judiciaires ainsi que des infrastructures judiciaires en mauvais état.

Partant de ce dysfonctionnement, Lubua (2005) a proposé dans sa recherche d'équiper les tribunaux afin que les autorités judiciaires puissent commencer à constituer des dossiers sur les cas des violences sexuelles et entamer des actions en justice au nom des victimes.

À cause de tous ces problèmes, les victimes n'ont pas confiance à la Justice, d'où le fait qu'elles recourent aux tribunaux coutumiers (à travers les 
arrangements à l'amiable) qui sont proches d'elles et qui utilisent une procédure connue par elles, car puisée dans la coutume. C'est le cas du «Lobé » dans le milieu traditionnel « Topoké », traduit par « Isonga Songa » dans le milieu urbain. Ainsi, le recours à la coutume permet aux parties d'obtenir une réparation du préjudice qu'il est difficile d'obtenir par voie judiciaire du fait de l'inexécution des jugements pour des causes diverses. Ainsi, l'étude a révélé que dans la Tshopo, le personnel judiciaire est buté à plusieurs problèmes dans l'exercice de son métier. Ceux-ci entravent la bonne marche de l'appareil judiciaire dans son ensemble. Comme conséquence, la voie du recours à l'arrangement à l'amiable devient fréquente.

Dans le même ordre d'idées, le BCNUDH (2014) avait conclu que dans de nombreuses régions de la RD. Congo, de nombreux cas de violence sexuelle font l'objet de règlement à l'amiable souvent perçus comme la plus efficace des procédures judiciaires. Malheureusement, celui-ci ne tient pas compte des intérêts de la victime. D'après la même source, dans beaucoup de cas, la famille de la victime, les agents de la Police Nationale Congolaise (PNC) et certaines autorités judiciaires encouragent la victime à trouver un arrangement avec l'agresseur, même lorsque celui-ci est un agent de l'État. Dans ce contexte, ce sont les chefs des familles des victimes et des auteurs qui mènent le règlement, écartant ainsi les victimes du processus. Dans certains cas, les agresseurs acceptent d'épouser les victimes pour leur éviter la stigmatisation dont elles seraient l'objet. Ainsi, ils peuvent échapper à des poursuites qui aboutiraient au paiement des amendes.

L'autre problème est celui de l'insuffisance et de l'éloignement géographique des juridictions. En RD. Congo, l'infraction de violence sexuelle est de la compétence des Tribunaux pour Enfants et de Grande Instance. Ainsi, la compétence matérielle des tribunaux s'étend à toute infraction punissable d'une peine de 5 ans de servitude pénale à la peine de mort. Pour rappel, l'infraction de violence sexuelle est punissable de 5 à 20 ans de prison. Lorsque cette dernière a entraîné la mort de la victime, elle est punissable d'une peine de servitude pénale à perpétuité (Article 170, loi sur les violences sexuelles en RD. Congo, 2006).

Cette étude a permis de comprendre que les Tribunaux pour Enfants et de Grande Instance habilités à instruire les dossiers des violences sexuelles sont insuffisants dans la Tshopo. Ils sont éloignés des justiciables qui habitent les milieux ruraux. En effet, les victimes des milieux ruraux qui désirent porter plainte en Justice doivent parcourir de longues distances, souvent à pied, pendant plusieurs jours et traverser des zones à haut risque d'insécurité. Ceci entraine un accès difficile des victimes aux tribunaux. La conséquence en est qu'elles sont découragées et se complaisent à des solutions coutumières, notamment les arrangements à l'amiable qui ne leur profitent pas. 
Les victimes ne bénéficient que d'un accès limité aux services juridiques, aux réparations et à l'indemnisation. Partant, il faut comprendre que si les soins complets (assistance médicale, psychologique, juridique et économique) sont disponibles dans certaines zones urbaines, ils demeurent cependant insuffisants et inadaptés ailleurs. Dans les zones isolées, où le système judiciaire est absent, les accords à l'amiable financier ou autres (y compris le mariage avec les agresseurs identifiés) sont des pratiques courantes, sans que la victime ne soit rétablie dans ses droits.

En troisième lieu, apparaît le problème de la plainte contre inconnu. Les résultats de l'étude ont révélé que certaines victimes des violences sexuelles ne connaissent pas leurs agresseurs, le cas des violences collectives et nocturnes. Si on ne connaît pas l'agresseur ou les agresseurs, on est obligé de porter plainte contre des « inconnus ». Il est vrai que le Parquet et la Police ont l'obligation de mener des enquêtes en cas de plainte contre des « inconnu », il se fait que, dans la plupart des cas, les plaintes contre des « inconnus» aboutissent rarement. Ceci n'encourage pas les victimes qui préfèrent garder silence, entrainant ainsi un impact négatif sur leur santé.

Un autre problème est le manque de confiance dans le système judiciaire et le recours fréquent au règlement à l'amiable ou coutumier. L'étude a permis de comprendre que le personnel judiciaire est buté à plusieurs difficultés qui ont entrainé le manque de confiance au système judiciaire par les victimes et leurs familles. Comme difficultés, l'étude cite les problèmes des infrastructures, l'irrégularité des salaires et le manque de formation nécessaire. Au-delà de celles-ci, il y a également l'insuffisance des magistrats. Par exemple, en 2013, le pays comptait environ 3.700 magistrats, ce qui équivalait alors à un magistrat civil pour 17.000 habitants (OSF, 2013). Ceci est aussi démontré par BCNUDH (2014) qui a révélé que le système judiciaire dispose d'un nombre insuffisant de magistrats et d'infrastructures capables de couvrir le vaste territoire de la RD. Congo.

Dans le même ordre d'idées, l'établissement des preuves par le personnel de la justice est un autre problème identifié. En matière pénale, la charge de la preuve revient à l'Officier du Ministère Public qui dispose des services des auxiliaires de la justice que sont les officiers de Police Judiciaire et les différents experts (psychologue et médecin) pour mener à bien sa tâche et obtenir la condamnation du prévenu.

En effet, l'établissement de la matérialité de l'infraction des violences sexuelles relève d'un véritable parcours du combattant, car les moyens de preuve en cette matière sont difficiles à obtenir. À titre d'exemple, pour les violences sexuelles commises loin du centre-ville, les Officiers de Police Judiciaire chargés de l'enquête ont souvent du mal à se rendre sur les lieux de l'infraction afin de procéder aux investigations. Les raisons avancées sont, entre autres, l'inaccessibilité à certains territoires et districts à cause des 
distances, de l'insécurité, de l'absence des infrastructures routières ou des moyens de déplacement.

En outre, la victime de la violence, habitée par un sentiment de honte et d'humiliation, hantée par la peur d'être rejetée par sa famille ou sa communauté, a tendance à se réfugier dans un silence faussement protecteur. Ce silence ne permet pas à l'Officier de Police Judiciaire de récolter les preuves dont il a besoin. Il est important de noter que la non féminisation du Corps des Officiers de Police Judiciaire crée une barrière pour la femme étant donné qu'elle a du mal à saisir la justice du fait que certaines victimes se sentent gênées de raconter la violence sexuelle subie aux personnes de sexe masculin. Ces femmes se sentent souvent incomprises, surtout lorsque l'Officier de Police Judiciaire, l'Officier du Ministère Public ou le juge l'interroge de manière assez brutale et directe, doutant même du fait qu'elle n'ait pas consenti aux relations sexuelles.

La collecte des preuves en matière des violences sexuelles pose également problème lorsqu'il s'agit de requérir l'avis d'un médecin pour constater les faits ; les médecins légistes étant absents et/ou peu nombreux. Du fait de cette carence, les victimes sont dirigées vers des médecins ordinaires qui les examinent et établissent, moyennant paiement, un certificat médical. Parfois, ces certificats sont incomplets et ne donnent donc pas tous les éléments dont le Juge a besoin pour déterminer s'il y a eu effectivement violence sexuelle. Devant cette situation, le Juge est contraint de classer le dossier sans suite ou d'envoyer le dossier incomplet en fixation au tribunal. À toutes ces difficultés s'ajoute parfois le fait que la situation est dénoncée trop longtemps après les faits ; ce qui profite au suspect, qui ne peut être condamné faute des preuves.

Le problème suivant est relatif à la poursuite des agresseurs par les instances judiciaires. Même si la poursuite des agresseurs est une tâche qui concerne directement la Police, les analyses faites ont montré que ce problème concerne particulièrement la victime. En effet, si l'agresseur n'est pas arrêté et traduit en Justice, la victime risque d'en souffrir, au risque d'impacter sa santé mentale et physique. En effet, la poursuite des agresseurs est réalisée par les instances judiciaires, après un premier travail d'arrestation réalisé par la Police Nationale Congolaise et le Parquet/Auditorat. Tout cas des violences sexuelles est dénoncé d'abord à la Police et au Parquet/Auditorat qui se saisissent du dossier et mettent sur pied un mécanisme pour arrêter l'auteur (ou les auteurs) de l'agression. Après l'analyse des matériaux, il est constaté que le premier obstacle ne facilitant pas le travail de la Police est la famille de l'agresseur qui le fait fuir.

Au-delà de cette attitude de la famille, l'étude a révélé qu'il y a des situations où c'est la communauté dans son ensemble qui fait face à la famille de la victime. Cette dernière se sentant dans l'insécurité, car vivant elle-même 
dans cette communauté, préfère se taire pour exploiter les pistes conduisant à des arrangements à l'amiable. Parfois, c'est la famille de la victime qui souhaite garder le secret car dans sa communauté, la violence sexuelle est considérée comme une malédiction ou un acte honteux. Ainsi, ces situations sont gérées en famille pour ne pas attirer l'attention du public.

Un autre problème est que la Police travaille et vit dans les conditions très difficiles. Ne disposant pas de moyen de transport adéquat, elle doit effectuer des longues distances à pieds, au besoin à l'aide des motos, recourant ainsi au service de taximans. Le paiement de ces derniers leur pose problème. Ce qui rend l'arrestation (la poursuite) de l'agresseur difficile.

$\mathrm{Au}$ vu de la misère dans laquelle les policiers sont plongés, certains agresseurs utilisent des astuces conduisant à des arrangements à l'amiable. Partant de ces arrangements, les agresseurs ont l'occasion de s'enfuir. Donc, dans la poursuite des agresseurs, les Policiers exposent également leur vie. Ainsi, il se dégage une certaine complicité (Policiers/agresseurs) ne permettant pas à certaines victimes de dénoncer les faits.

\section{Discussion des résultats}

Que retenir de l'analyse des résultats de cette étude qui a planché sur les problèmes liés au parcours juridique des victimes des violences sexuelles dans la province de la Tshopo ? Comme affirmé plus haut, les résultats de l'étude ont permis de comprendre que les problèmes rencontrés par les victimes des violences sexuelles lors de leur parcours juridique sont nombreux et de diverses natures. Tous ces problèmes décrits ci-haut, non seulement affectent la santé et la vie de la victime, mais aussi empêchent cette dernière à obtenir justice et réparation. Cette section va confronter les résultats obtenus dans cette étude, à ceux d'autres recherches menées sur la même thématique.

En effet, l'étude a montré, partant des résultats décrits ci-haut que les activités auxquelles les victimes des violences sexuelles se livrent les amènent à rentrer tardivement à la maison, à sillonner la ville et à fréquenter les lieux tels que les champs, les brousses et les forêts pour couper les bois, etc. Ces endroits constituent des lieux de prédilection pour le viol. Ces résultats concordent avec ceux qui sont obtenus par le Ministère du Genre, de la Famille et de l'Enfant de la RD. Congo (2013) qui confirment le fait que tous ces endroits exposent les femmes à la violence.

Cette étude a également révélé que la pauvreté est l'un des problèmes majeurs auxquels sont confrontées les victimes des violences sexuelles. C'est également ce qu'avait affirmé le Ministère du Genre, de la Famille et de l'Enfant de la RD. Congo (2013). Dans cette étude, il est fait mention de la pauvreté des femmes comme l'un des problèmes majeurs auxquels sont confrontées les victimes des violences sexuelles. Dans le même ordre d'idées, Lubua (2005) avait conclu que les victimes mènent une vie matérielle 
mauvaise ou médiocre. Comme si cela ne suffisait pas, l'éclatement des guerres successives à Kisangani a aggravé leur pauvreté.

Les résultats ont également relevé le rôle joué par les coutumes dans l'accroissement des violences sexuelles. En effet, dans une conférence de presse (MONUSCO, 2013), il a été clairement démontré que l'enracinement des coutumes, le silence de la plupart des victimes des violences sexuelles, la tendance au règlement à l'amiable des cas de viol et l'ignorance de la loi sur les violences sexuelles sont autant de facteurs qui ont favorisé l'ampleur de ces violences dans le district.

Un autre problème trouvé est la discrimination dont les victimes sont l'objet. Ceci rejoint la conclusion du rapport d'Amnesty International (2004) qui a montré que les victimes des violences sexuelles vivent dans une exclusion sociale et économique. Ce rapport a clairement dit que la discrimination à l'égard des victimes de viol est très répandue et que celles-ci sont souvent rejetées, voire insultées et menacées par leur entourage. Un grand nombre d'entre elles ont été brutalement abandonnées par leur mari et doivent, donc s'occuper seules de leurs enfants.

L'étude a démontré la lourdeur de la justice congolaise entrainant ainsi les procédures judiciaires coûteuses pour les victimes et le recours excessif aux coutumes par les communautés en cas des violences sexuelles. Ces résultats apparaissent également dans le rapport produit par le Réseau National des ONG pour le développement de la Femme (RENADEF, 30 Août 2019). En effet, celui-ci a révélé une certaine lourdeur dans l'appareil judiciaire. Ceci crée de la méfiance dans la population qui trouve que la Justice est très coûteuse et longue et que c'est mieux de recourir à la coutume pour les arrangements à l'amiable. D'après la conclusion dudit rapport, à cause de la lourdeur dans le fonctionnement de la Justice et faute des moyens financiers suffisants pour payer à la fois le service d'un avocat et les frais de procédures judiciaires, les familles des victimes préfèrent soit procéder aux arrangements à l'amiable, soit à l'abandon des causes à mi-chemin.

Parmi d'autres problèmes auxquels sont confrontés les victimes des violences sexuelles, l'étude a insisté sur le manque de confiance dans le système judiciaire, la carence ou l'insuffisance de juges et de magistrats femmes, l'inefficacité des tribunaux causée par le dysfonctionnement de ces derniers, le poids des frais en justice, les longues distances à parcourir par les victimes des violences sexuelles ainsi que la difficulté à apporter la preuve. Ces problèmes ont été également soulignés dans un rapport spécial présenté par Clifford et al. (2008).

Tel est le cas pour la présente étude, le BCNUDH (2014) avait permis d'identifier d'autres problèmes auxquels sont confrontées les victimes en RD. Congo, c'est notamment l'éloignement et l'insuffisance des Tribunaux, les représailles, le recours à l'arrangement à l'amiable et la pauvreté des victimes, 
les nombreuses lacunes dans les institutions judiciaires, le mariage forcé avec les agresseurs ainsi que la stigmatisation des victimes. Comme réponse à la première question soulevée à l'introduction, il y a lieu de retenir que les problèmes liés au parcours juridique des victimes des violences sexuelles sont à la fois d'ordre général et liés au dysfonctionnement de l'appareil judiciaire.

Concernant le manque de confiance dans le système judiciaire et le recours fréquent au règlement à l'amiable identifié parmi les problèmes des victimes des violences sexuelles, il y a lieu d'affirmer, partant des résultats de cette étude que la procédure coutumière basée sur l'arrangement à l'amiable ne privilégie pas l'intérêt de la victime. Ceci fait dire à Botima (2006) que la vie sexuelle de la femme est banalisée par la pratique coutumière, tout en spécifiant que la victime des violences sexuelles est discriminée par le recours aux pratiques coutumières. Azama (2006) avait conclu que les victimes sont stigmatisées. Au-delà de ces résultats concordants, les analyses réalisées dans cette étude ont permis de conclure que le recours à la coutume permet aux parties plaignantes d'obtenir une réparation du préjudice qu'il est difficile d'obtenir par voie judiciaire du fait de l'inexécution des jugements.

Le poids des coutumes, traditions et cultures comme un problème auquel sont butées les victimes des violences sexuelles est aussi souligné par Eca-Widnet (1999). Dans le même ordre d'idées, le recours aux pratiques coutumières comme favorisant les violences sexuelles est fustigé par plusieurs auteurs. Si Musafiri (2011) avait conclu que les violences sexuelles sont favorisées par les pratiques culturelles (coutumières) et que la femme est une victime passive qui n'a pas à s'opposer au diktat de la coutume, Mopongo (2006) a montré que la coutume a permis de mettre en relief certaines violences soutenues par la tradition dans le domaine de la vie sexuelle. Pour sa part, Kabasele (2006) a conclu que la pratique coutumière telle que l'initiation des filles et des garçons à la vie sexuelle est une dose du sadisme car, l'acte sexuel destiné à fournir du plaisir apparaît comme une douleur infligée à la femme.

La problématique des violences sexuelles étant complexe, l'étude l'a abordée dans une approche de la théorisation enracinée qui est l'une des approches indiquées pour étudier les phénomènes complexes. Les matériaux sont analysés de manière à minimiser les principaux reproches adressés à cette approche, notamment le manque de rigueur dans l'analyse des données, la mauvaise restitution du travail du chercheur dans sa recherche et la tension constante entre la créativité et la scientificité (Garreau \& Bandeira-De-Mello, 2008 ; 2011). Pour dépasser cette tension, l'étude s'inscrit dans le cadre proposé par Alversson et al. (2008) selon lequel la créativité et la scientificité peuvent se compléter, voire se renforcer dans la théorie enracinée.

Cette étude a des limites certaines. Il est vrai que les résultats obtenus, à travers les matériaux analysés ont permis de rendre compte des problèmes 
liés au parcours juridique des victimes des violences sexuelles dans la province de la Tshopo en RD. Congo. Cependant, il faut reconnaître que les résultats de cette étude ne sont pas à extrapoler à l'ensemble des provinces pour un pays à dimension continentale et qui regorge plus de 450 tribus. Ainsi, les résultats concernent essentiellement les populations impliquées et sur lesquelles les matériaux ont été collectés. En attendant les conclusions d'autres études dans d'autres contrées, retenons tout de même que les analyses faites dans cette étude ont permis de rendre compte de l'ampleur des principaux problèmes rencontrés par les victimes des violences sexuelles, aussi bien dans leur parcours juridique que dans leur vie quotidienne.

Partant de différents problèmes évoqués dans cette étude, il y a lieu de retenir que les résultats obtenus permettent à la fois une sensibilisation et un plaidoyer auprès de toutes les personnes de bonne volonté et de différents partenaires qui travaillent en faveur des victimes. Les actions concrètes, notamment celles que le CICR (2009) mène dans les provinces de Nord et Sud-Kivu, à savoir les campagnes de prévention et de réduction des risques en sensibilisant les autorités et les communautés à la problématique des violences sexuelles, doivent s'intensifier et être encouragées. La Justice devait être redynamisée de manière à lui permettre de décourager les auteurs des violences sexuelles, par l'application stricte et rigoureuse des lois en cette matière. De même, il est impérieux d'impliquer les communautés en général et en particulier les leaders communautaires dans le processus de lutte contre la stigmatisation des victimes des violences sexuelles et les préjugés qui sont souvent à l'origine de ces violences.

Le message de sensibilisation et de conscientisation devait se recentrer autour des thèmes en lien direct avec la problématique des violences sexuelles. Il est souhaitable de diversifier les méthodes en fonction du public cible et de sélectionner celui-ci en tenant compte de son degré de vulnérabilité aux violences sexuelles. L'étude se termine en disant qu'une bonne façon d'aider les victimes, c'est d'intensifier les cliniques juridiques qui seraient prises en charge financièrement par l'État congolais et par d'autres partenaires. Toutes ces implications issues des résultats de cette étude permettent de répondre à la deuxième question soulevée à l'introduction.

\section{Conclusion}

L'objectif majeur de cette étude était d'analyser les problèmes liés au parcours juridique des victimes des violences sexuelles dans la province de la Tshopo en RD. Congo. Pour atteindre cet objectif, plusieurs sources d'informations ont été prises en considération. À ce stade, il faut retenir que l'étude a identifié, à la fois des problèmes d'ordre général et des problèmes liés au dysfonctionnement de l'appareil judiciaire. Ces problèmes constituent dans leur majorité une entrave majeure au parcours juridique des victimes des 
violences sexuelles. La recherche a soulevé deux questions, à savoir 1) quels sont les différents problèmes que rencontrent les victimes de violences sexuelles tout au long de l'instruction en justice de leurs dossiers et, 2) quelles stratégies peut-on déployer pour élaguer ces problèmes et permettre une prise en charge efficace et équitable des victimes de ces violences ?

Parmi les problèmes d'ordre général que la recherche a identifié, il convient de mentionner le manque de moyens pour les victimes et leurs familles (une pauvreté ne leur permettant pas d'entreprendre une action en justice) et l'ignorance du droit, l'insécurité et la peur des représailles, le statut de la femme dans les coutumes, l'atteinte à la dignité de la victime et, l'environnement social dans lequel vivent les victimes des violences sexuelles. D'autres problèmes relevant du dysfonctionnement de l'appareil judiciaire et qui entravent le parcours juridique des victimes des violences sexuelles restent le dysfonctionnement des services judiciaires (les manœuvres dilatoires, c'està-dire le fait pour les juges et les magistrats de repousser perpétuellement les audiences pour freiner le procès ou bloquer l'action par des procédures irrégulières, la lenteur ou la lourdeur des procédures dans l'instruction des dossiers, l'ignorance de la loi en matière des violences sexuelles par certains magistrats), le fait que pour certains dossiers, la procédure judiciaire donne difficilement la réponse en ce qui concerne la réparation civile, bien que le prévenu ait été condamné pénalement, l'injustice ou la partialité de certains juges, l'insuffisance et l'éloignement géographique des juridictions compétentes en matière des violences sexuelles, la plainte contre des inconnus et la non-exécution des jugements existants, sont autant des problèmes entravant le parcours juridique des victimes des violences sexuelles dans la province de la Tshopo en RD. Congo.

Pour terminer, retenons que l'accès à la Justice dans la province de la Tshopo en RD. Congo est très difficile pour les victimes des violences sexuelles du fait de leur statut socio-économique défavorable. Confrontées à la crainte de leurs bourreaux, au manque de connaissance des voies de recours, au paiement de certains frais en justice que les victimes considèrent comme étant exorbitants et aux difficultés de transport pour atteindre les Tribunaux compétents, ces dernières éprouvent d'énormes difficultés pour que justice leur soit rendue. Même si elles y parviennent, les décisions de justice prononcées ne sont pas appliquées. Que faire ? Il faut donc une sensibilisation et une implication de tous, tout en menant des actions concrètes pour effrayer les agresseurs. Donc, la solution passe obligatoirement par une action conjuguée de tous. 


\section{References:}

1. Alversson, Hardy \& Harley. (2008). Reflecting on Reflexivit: Reflexive Textual Pratices in Organization and Management Theory. Journal of Management Studies.45(3),480-501.

2. Amnesty International. (2004). République Démocratique du Congo : violences sexuelles : un urgent besoin de réponses adéquates. Bulletin d'information 257/2004.

3. Azama, P. (2006). Les problèmes affectifs de quelques femmes \& filles victimes des violences sexuelles à Kisangani. [Mémoire de licence, Université de Kisangani]. Kisangani.

4. Beck, C.T. (1999). Grounded theory research. Dans Fain, J.A (Ed.), Reading, Understanding and Applying Nursing Research, 205-225. Philadelpjia. PA: F.A.Davis.

5. Botima, C. (2006). Les bases culturelles de violences sexuelles en R.D.Congo: cas de l'éthnie Lokele dans la Province Orientale. [Mémoire de licence, Université de Kisangani]. Kisangani.

6. Bureau Conjoint des Nations Unies aux Droits de l'Homme (MONUSCO/HCDH) en République démocratique du Congo. (2014). Nations Unies : Droits de l'homme.

7. Bryant, A. et Charmaz, K. (2007). The Sage Handbook of Grounded Theory. Thousand Oaks: Sage Publications.

8. Charmaz, K. (2006). Constructing Grounded Theory. A Practical Guide through Qualitative Analysis. Thousand Oaks: Sage Publications.

9. CICR, (2009). Violences sexuelles en République Démocratique du Congo: prévention et aide aux victimes. Consulté le 17 juin 2021 sur https://www.icrc.org>congo-Kinshasa-interview- 020309

10. Clifford, L., Eichstaedt, P., Glassborow, K., Goetze, K. et Ntiryica, C. (2008). Violence sexuelle en République Démocratique du Congo. The Netherlands: Institute for War \& Peace

11. Reporting. Consulté le 17 juin 2021 sur www.iwpr.net

12. Corbin, J. et Strauss, A. (2008). Basics of Qualitative Research. Thousand Oaks: Sage Publications.

13. Dey, I. (1999). Grounding Grounded Theory: Guidelines for Qualitative Inquiry. San Diego, CA: Kluwer.

14. Duckaerts, F. (1969). La formation du lien sexuel. Bruxelles : Ed. Charles Dessart.

15. Eca-Widnet. (1999). La violence sexuelle contre la femme, manuel pour l'animation (Traduit de l'anglais violence against women by Anne Marie Mpundu, 2001). Kinshasa : Ed. Filles de S-Paul.

16. Focobiderco. (2019). Etude des coûts de la prise en charge juridique et judiciaire des VVS dans la ville de Kisangani. Kisangani. 
17. Garreau, L. et Bandeira-De-Mello, R. (Mai 2008). Possibilités et pièges liés à l'utilisation des logiciels dans le processus d'analyse au travers de la théorie enracinée. [Conférence AIMS.] Sophia Antipolis, France. https://halshs.archives-ouvertes.fr/halshs-00339851

18. Garreau, L. et Bandeira-De-Mello, R. (2010). La théorisation enracinée en pratique: vers un dépassement de la tension entre scientificité et créativité dans les recherches basées sur la théorie enracinée ? AIMS, 1-19. Luxembourg. https://halshs.archives-ouvertes.fr/halshs-00580543.

19. Glaser, B.G. et Strauss, A.L. (1967). The Discovery of Grounded Theory: Strategies for Qualitative Research. New York: Aldine.

20. Glaser, B.G. (1995). A Look at Grounded Theory: 1984 to 1994. Dans Glaser, B.G. (Ed.),

21. Grounded Theory: 1984-1994, (3-17). Mill Valey, CA: Sociolog Press.

22. Glaser, B.G. (1998). Doing Grounded Theory. Mill Valley. CA: Sociology Press.

23. Glaser, B.G. (2001). The Grounded Theory Perspective: Conceptualization Contrasted with Description. Mill Valey. CA: Sociology Press.

24. Guillemette, F. (2006). L'approche de la Grounded Theory; pour innover? Recherches Qualitatives, 26 (1), 32-50. Consulté le 22 sur http://www.recherche- qualitative.qc.ca/Revue.html

25. Jacques, M-C, Maude, H., Gallagher, F., St-Cyr Tribble, D. (2014). La théorisation ancrée.Une théorisation ancrée pour l'étude de la transition des perceptions de l'état de santé.

26. In La Recherche en Sciences Humaines, Sociales et de la Santé. Quebec : Presses de 1'Université du Quabec, 97-116.

27. Clifford, L. ; Eichstaedt, P. ; Glassborow, K. ; Goetze, K. et Ntirica, C. (2008). Violence sexuelle en République Démocratique du Congo. Institute for War \& Peace Reporting. The Netherlands, www.ipr.net

28. CDF. (2017, 2 juillet). Les victimes des violences sexuelles encouragées à saisir la justice. [Atelier ]. Kisangani. https://www.radiookapi.net>actualité>société>Kisangani.

29. CVS, INNSSEE-ONDRP (2013). Rapport d'enquête sur le cadre de vie et sécurité.

https://www.memoiretraumatique.org-fils

30. Kabasele, D. (2006). Les bases culturelles de violences sexuelles en RD.Congo : cas de l'éthnie Lulua du Kasai Occidental. [Mémoire de licence, Université de Kisangani]. Kisangani.

31. Laperrière, A. (1997). «La théorisation ancrée (grounded theory): démarche analytique et comparaison avec d'autres approches 
apparentées». Dans Poupart, J., Deslauriers, J-P, Groulx, L.-H. Laperrière, A, Mayer, R. et Pires, A.P. (dir.), La recherche qualitative :enjeux épistémologiques et méthodologiques. Boucherville: Gaëtan Morin, 309-340.

32. Loi ${ }^{\circ} 06 / 018$ du 20 juillet (2006) modifiant et complétant le Décret du 30 janvier 1940 portant Code pénal congolais.

33. Luckerhoff, J. et F. Guillemette (dir.) (2013). Méthodologie de la théorisation enracinée. Québec: Presses de l'Université du Québec.

34. Lubua, A. (2005). Impact des violences sexuelles sur la santé mentale des femmes et jeunes filles victimes dans la ville de Kisangani. [Mémoire de licence, Université de Kisangani]. Kisangani.

35. Maertens de Noordhout, F. (2013). Violences sexuelles en République Démocratique du Congo : Mais que fait la police ? Un état de non-droit à la recherche d'un système normatif. Le cas d'EUPOL RD Congo. Revue interdisciplinaire d'études juridiques. (71), 213-241.

36. https://www.cain.info/revue- interdisciplinaire-d-etudes-juridiques2013-2-page-213.htm

37. May, K.A. (1996). Diffusion, dilution, or distillation ?: The case of grounded theory method. Qualitative Health Research, 6 (3), 309-311.

38. Mopongo, P. (2006). Les bases culturelles des violences sexuelles en RD.Congo. [Mémoire de licence, Université de Kisangani]. Kisangani.

39. Morse, J.M \& Richards, L. (2002). Readme first. Thousand Oaks, CA: Sage.

40. MONUSCO (2013, 9 octobre). Radio Okapi : Les violences sexuelles commises en République Démocratique du Congo. [Conférence de Presse]. Kinshasa.

41. Musafiri, V. (2011). Influence de la culture sur la violence sexuelle: enquête menée au sein de la tribu Fuliiru dans le territoire d'Uvira en RD.Congo. [Mémoire de licence, Université de Kisangani]. Kisangani.

42. Norton, L. (1999). The philosophical bases of grounded theory and their implications for research pratice. Nurse Researcher, 7 (1), 31-43.

43. Peterman, A. et al. (2011). Estimates and Determinants of Sexual Violence Against Women in the Democratic Republic of Congo. In American Journal of Public Health. 1060.

44. Strauss, A. et J. Corbin. (1998). Basics of Qualitative Research Thousand Oaks. Sage Publications.

45. Tremblay, N. (2005). La qualité de l'intimité chez les agresseurs sexuels intrafamiliaux [Mémoire de Master, Université du Quebec à Chicoutimi]. Quebec.

46. Ombiabo, A. (2020). Etude des traumatismes psychiques chez les femmes et filles victimes de violences sexuelles dans un contexte de 
paix à Kisangani dans la province de la Tshopo en République Démocratique du Congo. [Mémoire de Fin de cycle, Université de Kisangani]. Kisangani.

47. OMS, UNSD, Office des Nations Unies contre la drogue. (2021, 9 mars). Une femme sur trois dans le monde est victime de violence. [Communiqué]. Geneve, New-York, https://www.who.int > ... > item. ...,

48. Ordonez, J. (2007). Exigences juridiques nationales : Poursuite des violences sexuelles et sexistes en République Démocratique du Congo. Ratification, mise en œuvre et coopération. Case Matrix Networt.

49. OSF (2013, juillet). Rapport de juille. République Démocratique du Congo.

50. RENADEF. (2019, août). Assistance juridique et judiciaire des VVS. Kisangani.

51. Société civile. (2019, mars). Déclaration de la Société civile sur la violence sexuelle.

[Déclaration].Kinshasa. https://4genderjustice.org>2019/10

52. Vasseur, A. Bernardo, A. Melo-Pinzon, G. et Musafiri Masika, E. (2019, avril). Evaluation conjointe des programmes de lutte contre les violences sexuelles en République Démocratique du Congo2005-2017. UNFPA.

53. UNFPA. (2013). Ampleur des violences sexuelles en République Démocratique du Congo et actions de lutte contre le phénomène de 2011 à 2012. Consulté le 21 juillet 2021 sur http://www.unfpa.org/webdav/site/global/shaered/documents/news20 13/Rapport\%20DM\%20SGBV\%202011-2012.pdf

54. Willing, C. (2001). Introducing Qualitative Research in Psychology. Buckingham, UK: Open University Press. 